University of Texas at El Paso

ScholarWorks@UTEP

\title{
$5-2013$
}

\section{Processing Quantities with Heavy-Tailed Distribution of Measurement Uncertainty: How to Estimate the Tails of the Results of Data Processing}

\author{
Michal Holčapek \\ University of Ostrava, Michal.Holcapek@osu.cz \\ Vladik Kreinovich \\ The University of Texas at El Paso, vladik@utep.edu
}

Follow this and additional works at: https://scholarworks.utep.edu/cs_techrep

Part of the Computer Sciences Commons

Comments:

Technical Report: UTEP-CS-13-32a

To appear in Proceedings of 3rd World Conference on Soft Computing, San Antonio, December 15-18, 2013.

\section{Recommended Citation}

Holčapek, Michal and Kreinovich, Vladik, "Processing Quantities with Heavy-Tailed Distribution of Measurement Uncertainty: How to Estimate the Tails of the Results of Data Processing" (2013). Departmental Technical Reports (CS). 771.

https://scholarworks.utep.edu/cs_techrep/771

This Article is brought to you for free and open access by the Computer Science at ScholarWorks@UTEP. It has been accepted for inclusion in Departmental Technical Reports (CS) by an authorized administrator of ScholarWorks@UTEP. For more information, please contact Iweber@utep.edu. 


\title{
Processing Quantities with Heavy-Tailed Distribution of Measurement Uncertainty: How to Estimate the Tails of the Results of Data Processing
}

\author{
Michal Holčapek ${ }^{1}$ and Vladik Kreinovich ${ }^{2}$ \\ ${ }^{1}$ Centre of Excellence IT4Innovations, University of Ostrava, Institute for Research \\ and Applications of Fuzzy Modeling, Ostrava, Czech Republic, \\ michal.holcapek@osu.cz \\ ${ }^{2}$ University of Texas at El Paso, El Paso, TX 79968, USA, vladik@utep.edu
}

\begin{abstract}
Measurements are never absolutely accurate; so, it is important to estimate how the measurement uncertainty affects the result of data processing. Traditionally, this problem is solved under the assumption that the probability distributions of measurement errors are normal - or at least are concentrated, with high certainty, on a reasonably small interval. In practice, the distribution of measurement errors is sometimes heavy-tailed, when very large values have a reasonable probability. In this paper, we analyze the corresponding problem of estimating the tail of the result of data processing in such situations.
\end{abstract}

\section{Formulation of the Problem}

Need for data processing. In many practical situations, we are interested in the values of a quantity $y$ which is not easy (or even impossible) to measure directly: for example, we may be interested in tomorrow's weather, in the distance to a faraway planet, in the amount of oil in an oil well, etc. In such situations in which we cannot measure $y$ directly, we can often measure $y$ indirectly, i.e.:

- measure the values of auxiliary quantities $x_{1}, \ldots, x_{n}$ which are related to the desired quantity $y$ by a known relation $y=f\left(x_{1}, \ldots, x_{n}\right)$, and then

- use the results $\widetilde{x}_{1}, \ldots, \widetilde{x}_{n}$ of measuring the quantities $x_{i}$ and the known dependence to compute the estimate $\widetilde{y}=f\left(\widetilde{x}_{1}, \ldots, \widetilde{x}_{n}\right)$ for $y$.

The process of computing $\widetilde{y}=f\left(\widetilde{x}_{1}, \ldots, \widetilde{x}_{n}\right)$ is known as data processing.

Need to estimating uncertainty of the result of data processing. Measurements are never $100 \%$ accurate; so, in general, the measurement results $\widetilde{x}_{i}$ are somewhat different from the actual values $x_{i}$ of the corresponding quantities. Because of these measurement errors, the estimate $\widetilde{y}=f\left(\widetilde{x}_{1}, \ldots, \widetilde{x}_{n}\right)$ is, in general, different from the desired value $y=f\left(x_{1}, \ldots, x_{n}\right)$ (often, there is an additional difference cause by the fact that the dependence between $y$ and $x_{i}$ is only approximately 
known). It is therefore important not just to generate an estimate $\widetilde{y}$, but also to gauge how much the actual value $y$ can differ from this estimate, i.e., what is the uncertainty of the result of data processing; see, e.g., [7].

Estimating uncertainty of the result of data processing: traditional statistical approach. Usually, there are many different (and independent) factors which contribute to the measurement error. In many such situations, it is possible to apply the Central Limit Theorem (see, e.g., [9]), according to which, under reasonable conditions, the distribution of the joint effect of numerous independent factors is close to normal. In such situations, it is therefore reasonable to assume that all the measurement errors $\Delta x_{i} \stackrel{\text { def }}{=} \widetilde{x}_{i}-x_{i}$ are independent and normally distributed.

To describe a normal distribution, it is sufficient to know the mean $\mu$ and the standard deviation $\sigma$. Thus, under the normality assumption, to gauge the distribution of each measurement error $\Delta x_{i}$, we must know the mean $\mu_{i}$ and the standard deviation $\sigma_{i}$ of this measurement error. If the known mean is different from 0 , this means that this measuring instrument has a bias; we can always compensate for this bias by subtracting the value $\mu_{i}$ from all the measured values. After this subtraction, the mean error will become 0 . Thus, without losing generality, we can assume that each measurement error is normally distributed with mean 0 and known standard deviation $\sigma_{i}$.

The traditional way of estimating the resulting uncertainty $\Delta y \stackrel{\text { def }}{=} \widetilde{y}-y$ in $y$ is based on this assumption. Specifically, since the measurement errors $\Delta x_{i}$ are usually relatively small, we can expand the expression

$$
\begin{gathered}
\Delta y=\widetilde{y}-y=f\left(\widetilde{x}_{1}, \ldots, \widetilde{x}_{n}\right)-f\left(x_{1}, \ldots, x_{n}\right)= \\
f\left(\widetilde{x}_{1}, \ldots, \widetilde{x}_{n}\right)-f\left(\widetilde{x}_{1}-\Delta x_{1}, \ldots, \widetilde{x}_{n}-\Delta x_{n}\right)
\end{gathered}
$$

in Taylor series in $\Delta x_{i}$, ignore quadratic and higher order terms, and keep only terms in $\Delta x_{i}$ in this dependence. As a result, we get an expression

$$
\Delta y=\sum_{i=1}^{n} c_{i} \cdot \Delta x_{i}
$$

where $c_{i} \stackrel{\text { def }}{=} \frac{\partial f}{\partial x_{i}}$. Based on this expression, we conclude that the linear combination $\Delta y$ of $n$ independent normally distributed random variables is also normally distributed, its mean value of is 0 , and its variance $\sigma^{2}$ is equal to: $\sigma^{2}=\sum_{i=1}^{n} c_{i}^{2} \cdot \sigma_{i}^{2}$ (see, e.g., [7]).

Heavy-tailed distributions. There are many practical situations in which the probability distribution for the measurement error is drastically different from normal. In many such situations, the variance is infinite; such distributions are called heavy-tailed. Since then, similar heavy-tailed distributions have been empirically found in many other application areas; see, e.g., $[1,8]$. 
Historical comment: heavy-tailed distributions and fractals. Heavy-tailed distributions surfaced in the 1960s, when Benoit Mandelbrot, the author of fractal theory, empirically studied the fluctuations of prices and showed [4] that largescale fluctuations follow the Pareto power-law distribution, where for some $x_{0}$, for all $x \geq x_{0}$, the probability density function has the form $\rho(x)=A \cdot x^{-\alpha}$, for some empirical constants $A>0$ and $\alpha \approx 2.7$. For this empirical value $\alpha$, variance is infinite.

Mandelbrot studied not only the local price fluctuations, but also the global geometry of the curves describing the dependence of price on time. It turned out that this analysis is closely related to the notion of dimension. Indeed, for sets $S$ which are smooth curves and surfaces and for volumes surrounded by smooth surfaces, dimension can be described as follows. For each $\varepsilon>0$, we can $\varepsilon$-approximate the set $S$ by a finite set $S^{\prime}=\left\{s_{1}, \ldots, s_{n}\right\}, \varepsilon$-approximate in the sense that:

- every point $s$ from the set $S$ is $\varepsilon$-close to some point $s_{i} \in S^{\prime}$, and

- vice versa, every point $s_{i} \in S^{\prime}$ is $\varepsilon$-close to some point $s \in S$.

For each set $S$, we can have $\varepsilon$-approximating sets $S^{\prime}$ with different number of elements. For each $\varepsilon$, we can gauge the size of the given set $S$ by finding the number of elements $N_{\varepsilon}(S)$ in the smallest $\varepsilon$-approximating finite set.

For a 1-D smooth curve $S$, the smallest number $N_{\varepsilon}(S)$ is attained if we take the points $s_{1}, \ldots, s_{n} \in S$ located at equal distance $\approx 2 \varepsilon$ from each other. The number of such points is asymptotically equal to $N_{\varepsilon}(S) \sim$ const $\cdot \frac{L}{\varepsilon}$, where $L$ is the length of the curve $S$.

For a 2-D smooth surface $S$, the smallest number $N_{\varepsilon}(S)$ is attained if we take the points on a rectangular 2-D grid with linear step $\approx \varepsilon$. The number of such points is asymptotically equal to $N_{\varepsilon}(S) \sim$ const $\cdot \frac{A}{\varepsilon^{2}}$, where $A$ is the area of the surface $S$.

For a 3-D body $S$, the smallest number $N_{\varepsilon}(S)$ is attained if we take the points on a rectangular 3 -D grid with linear step $\approx \varepsilon$. The number of such points is asymptotically equal to $N_{\varepsilon}(S) \sim$ const $\cdot \frac{V}{\varepsilon^{3}}$, where $V$ is the volume of the 3-D body $S$.

It turns out that for the price trajectory $S$, we have $N_{\varepsilon}(S) \sim \frac{C}{\varepsilon^{a}}$ for some constant $C$ and a fraction (non-integer) $a$. By analogy with the smooth sets, the value $a$ is called a dimension of the trajectory $S$. Thus, the trajectory $S$ is a set of a fractal dimension; Mandelbrot called such sets fractals.

The above empirical result, together with similar empirical discovery of heavy-tailed laws in other application areas, has led to the formulation of fractal theory; see, e.g., $[5,6]$.

\section{Comments.}

- Please note that Mandelbrot's empirical observations only describe the probability density $\rho(x)$ for values $x \geq x_{0}$; the values $\rho(x)$ for $x<x_{0}$ can be different. 
- In general, the condition that $\int \rho(x) d x=1$ implies that $\alpha>1$.

- One can easily check that the variance $\int x^{2} \cdot \rho(x) d x$ is infinite when $\alpha \leq 3$.

Problem. If the measurement errors $\Delta x_{i}$ of the inputs $x_{i}$ are distributed according to the heavy-tailed distributions, then what can we conclude about $\Delta y$ ?

What we do in this paper. In this paper, we provide an answer to the above question for the simplest cases when data processing consists of applying a single arithmetic operation: addition, subtraction, multiplication, or division.

\section{Main Results}

Case of addition $y=f\left(x_{1}, x_{2}\right)=x_{1}+x_{2}$. For addition, $\Delta y=\Delta x_{1}+\Delta x_{2}$. When the measurement error $\Delta x_{1}$ of the first input has a tail with asymptotics $\rho_{1}\left(\Delta x_{1}\right) \sim A_{1} \cdot\left|\Delta x_{1}\right|^{-\alpha_{1}}$ and the measurement error $\Delta x_{1}$ of the first input has a tail with asymptotics $\rho_{2}\left(\Delta x_{2}\right) \sim A_{2} \cdot\left|\Delta x_{2}\right|^{-\alpha_{2}}$, then the tail for $\Delta y$ has the asymptotics $\rho(\Delta y) \sim A \cdot|\Delta y|^{-\alpha}$ with $\alpha=\min \left(\alpha_{1}, \alpha_{2}\right)$.

Proof for the case of addition $y=f\left(x_{1}, x_{2}\right)=x_{1}+x_{2}$. We know that $\rho(\Delta y)=$ $\int \rho_{1}\left(\Delta x_{1}\right) \cdot \rho_{2}\left(\Delta y-\Delta x_{1}\right) d\left(\Delta x_{1}\right)$. Asymptotics mean that for any given accuracy, for sufficiently large values $\Delta x_{1}$ and $\Delta x_{2}$, we have $\rho_{1}\left(\Delta x_{1}\right) \approx A_{1} \cdot\left|\Delta x_{1}\right|^{-\alpha_{1}}$ and $\rho_{2}\left(\Delta x_{2}\right) \approx A_{2} \cdot\left|\Delta x_{2}\right|^{-\alpha_{2}}$. What is the asymptotic expression for the probability density $\rho(\Delta y)$ for large values $\Delta y$ ?

A large value of $\Delta y=\Delta x_{1}+\Delta x_{2}$ can come from three different situations:

1) when $\Delta x_{1}$ is large (i.e., the asymptotic expression for $\rho_{1}\left(\Delta x_{1}\right)$ holds) and $\Delta x_{2}$ is not large in this sense;

2) when $\Delta x_{2}$ is large (i.e., the asymptotic expression for $\rho_{2}\left(\Delta x_{2}\right)$ holds) and $\Delta x_{1}$ is not large in this sense; and

3) when both $\Delta x_{1}$ and $\Delta x_{2}$ are large in this sense.

The first situation leads to terms proportional to $\left|\Delta x_{1}\right|^{-\alpha_{1}}=\left|\Delta y-\Delta x_{2}\right|^{-\alpha_{1}}$. Since in this case, $\Delta x_{2}$ is limited by the threshold after which the values become large, we have $\Delta x_{2} / \Delta y \rightarrow 0$ as $\Delta y \rightarrow \infty$ and thus, $\left|\Delta y-\Delta x_{2}\right|^{-\alpha_{1}} \sim|\Delta y|^{-\alpha_{1}}$. The second situation similarly leads to terms asymptotically equal to $|\Delta y|^{-\alpha_{2}}$.

In the third case, for some $K>1$, the integral which describes $\rho(\Delta y)$ (over the whole real line) can be represented as a sum of the integral $I_{\text {in }}(\Delta y)$ over $[-K \cdot|\Delta y|, K \cdot|\Delta y|]$ and the integral $I_{\text {out }}(\Delta y)$ over the outside of this interval.

The inner integral $I_{\text {in }}(\Delta)$ is bounded by $M \cdot(2 K \cdot|\Delta y|)$, where $M$ is the maximum of the the product

$$
\rho_{1}\left(\Delta x_{1}\right) \cdot \rho_{2}\left(\Delta y-\Delta x_{1}\right)=A_{1} \cdot\left(\Delta x_{1}\right)^{-\alpha_{1}} \cdot A_{2} \cdot\left(\Delta y-\Delta x_{1}\right)^{-\alpha_{2}} .
$$

Differentiating this expression w.r.t. $\Delta x_{1}$ and equating derivative to 0 , we conclude that $\Delta x_{1}=\frac{\alpha_{1}}{\alpha_{1}+\alpha_{2}} \cdot \Delta y$, hence the corresponding maximum is equal to const $\cdot|\Delta y|^{-\left(\alpha_{1}+\alpha_{2}\right)}$. Thus,

$$
I_{\text {in }}(\Delta y) \leq M \cdot(2 K \cdot|\Delta y|)=\text { const } \cdot|\Delta y|^{-\left(\alpha_{1}+\alpha_{2}-1\right)}
$$


for some positive constant.

Outside the interval, $|\Delta y| \leq \frac{1}{K} \cdot\left|\Delta x_{1}\right|$, thus, $\left|\Delta y-\Delta x_{1}\right| \leq\left(1+\frac{1}{K}\right) \cdot\left|\Delta x_{1}\right|$ and so,

$$
\begin{gathered}
\rho_{1}\left(\Delta x_{1}\right) \cdot \rho_{2}\left(\Delta y-\Delta x_{1}\right)=A_{1} \cdot\left|\Delta x_{1}\right|^{-\alpha_{1}} \cdot A_{2} \cdot\left|\Delta y-\Delta x_{1}\right|^{-\alpha_{2}} \leq \\
A_{1} \cdot A_{2} \cdot\left(1+\frac{1}{K}\right)^{-\alpha_{2}} \cdot\left|\Delta x_{1}\right|^{-\alpha_{1}} \cdot\left|\Delta x_{1}\right|^{-\alpha_{2}}=\text { const } \cdot\left|\Delta x_{1}\right|^{-\left(\alpha_{1}+\alpha_{2}\right)}
\end{gathered}
$$

for some positive constant. Integrating both sides of the resulting inequality, we conclude that

$$
\begin{gathered}
I_{\text {out }} \leq \int_{-\infty}^{-K \cdot|\Delta y|} \text { const } \cdot\left|\Delta x_{1}\right|^{-\left(\alpha_{1}+\alpha_{2}\right)} d\left(\Delta x_{1}\right)+ \\
\int_{K \cdot|\Delta y|}^{\infty} \operatorname{const} \cdot\left|\Delta x_{1}\right|^{-\left(\alpha_{1}+\alpha_{2}\right)} d\left(\Delta x_{1}\right)=\text { const } \cdot|\Delta y|^{-\left(\alpha_{1}+\alpha_{2}-1\right)}
\end{gathered}
$$

for some positive constant.

Both $I_{\text {in }}(\Delta y)$ and $I_{\text {out }}(\Delta y)$ are bounded by const $\cdot|\Delta y|^{-\left(\alpha_{1}+\alpha_{2}-1\right)}$, so their sum $\rho(\Delta y)$ is also bounded by a similar expression.

Summarizing: the asymptotic expression for $\rho(\Delta y)$ is the sum of three positive terms of the type $|\Delta y|^{-\alpha}$ : a term corresponding to $\alpha=\alpha_{1}$, a term corresponding to $\alpha=\alpha_{2}$, and a term bounded by $\alpha=\alpha_{1}+\alpha_{2}-1$. Since $\alpha_{i}>1$, we have $\alpha_{1}+\alpha_{2}-1>\alpha_{i}$.

In general, when $\alpha<\alpha^{\prime}$, then for large $z$, the ratio $\frac{z^{-\alpha^{\prime}}}{z^{-\alpha}}$ tends to 0 . This means in the sum of power-law asymptotic expressions, the term with the smallest value of $\alpha$ dominates, in the sense that the asymptotics of the sum follows the power law with the smallest possible exponent $\alpha$. In our case, since $\alpha_{1}+\alpha_{2}-1>\alpha_{i}$, this smallest exponent is $\min \left(\alpha_{1}, \alpha_{2}\right)$.

Case of a general linear combination. One can check that a similar formula holds for the difference $y=x_{1}-x_{2}$ and, more generally, for an arbitrary linear combination $y=a_{0}+\sum_{i=1}^{m} a_{i} \cdot x_{i}$. Namely, when the measurement error $\Delta x_{i}$ of the the $i$-th input has a tail with asymptotics $\rho_{i}\left(\Delta x_{i}\right) \sim A_{i} \cdot\left|\Delta x_{i}\right|^{-\alpha_{i}}$, then the tail for $\Delta y$ has the asymptotics $\rho(\Delta y) \sim A \cdot|\Delta y|^{-\alpha}$ with $\alpha=\min \left(\alpha_{1}, \ldots, \alpha_{m}\right)$.

Case of product $y=f\left(x_{1}, x_{2}\right)=x_{1} \cdot x_{2}$ : analysis of the problem. For the product, from $y=x_{1} \cdot x_{2}$ and $y+\Delta y=\widetilde{y}=\widetilde{x}_{1} \cdot \widetilde{x}_{2}=\left(x_{1}+\Delta x_{1}\right) \cdot\left(x_{2}+\Delta x_{2}\right)$, we conclude that $\Delta y=\Delta x_{1} \cdot x_{2}+x_{1} \cdot \Delta x_{2}+\Delta x_{1} \cdot \Delta x_{2}$.

We know the asymptotics of the probability distribution for $\Delta x_{1}$ and $\Delta x_{2}$, so $\Delta x_{1} \cdot x_{2}$ and $x_{1} \cdot \Delta x_{2}$ should have asymptotics with the same exponents $\alpha_{1}$ and $\alpha_{2}$. Let us find the asymptotics for the product $r \stackrel{\text { def }}{=} \Delta x_{1} \cdot \Delta x_{2}$. Similarly to the case of addition, the corresponding terms come from three cases: 
- when $\Delta x_{1}$ is large and $\Delta x_{2}$ is not large; this leads to terms $\sim|r|^{-\alpha_{1}}$;

- when $\Delta x_{2}$ is large and $\Delta x_{1}$ is not large; this leads to term $\sim|r|^{-\alpha_{2}}$;

- when both $\Delta x_{1}$ and $\Delta x_{2}$ are large; this leads to the term $\sim|r|^{-\left(\alpha_{1}+\alpha_{2}-1\right)}$, which (similarly to the case of addition) can be asymptotically ignored in comparison with terms $\sim|r|^{-\alpha_{i}}$.

Thus, similarly to the case of addition, we have terms with exponent $\alpha_{1}$, we have terms with exponent $\alpha_{2}$, and we have other terms which can be asymptotically ignored. Hence, we arrive at the following conclusion.

Case of product $y=f\left(x_{1}, x_{2}\right)=x_{1} \cdot x_{2}$ : result. When the measurement error $\Delta x_{1}$ of the first input has a tail with asymptotics $\rho_{1}\left(\Delta x_{1}\right) \sim A_{1} \cdot\left|\Delta x_{1}\right|^{-\alpha_{1}}$ and the measurement error $\Delta x_{2}$ of the second input has a tail with asymptotics $\rho_{2}\left(\Delta x_{2}\right) \sim A_{2} \cdot\left|\Delta x_{2}\right|^{-\alpha_{2}}$, then $\rho(\Delta y) \sim A \cdot|\Delta y|^{-\alpha}$ with $\alpha=\min \left(\alpha_{1}, \alpha_{2}\right)$.

Case of product or ratio of several terms. One can check that a similar formula holds for the ratio $y=x_{1} / x_{2}$ and, more generally, for an arbitrary combination $y=a_{0} \cdot \prod_{i=1}^{m} x_{i}^{a_{i}}$. Namely, when the measurement error $\Delta x_{i}$ of the the $i$-th input has a tail with asymptotics $\rho_{i}\left(\Delta x_{i}\right) \sim A_{i} \cdot\left|\Delta x_{i}\right|^{-\alpha_{i}}$, then the tail for $\Delta y$ has the asymptotics $\rho(\Delta y) \sim A \cdot|\Delta y|^{-\alpha}$ with $\alpha=\min \left(\alpha_{1}, \ldots, \alpha_{m}\right)$.

Comment. The main objective of this paper is to deal with measurement (epistemic) uncertainty. However, the same formula can be used if we have aleatory uncertainty. For example, we can use these formulas to analyze what happens if:

- we have a population of two-job individuals with first-salary distribution $\rho_{1}\left(x_{1}\right)$ and second-salary distribution $\rho_{2}\left(x_{2}\right)$,

- we know that these distributions are independent, and

- we want to find the distribution of the total salary $y=x_{1}+x_{2}$.

\section{Future Work: From Asymptotics to a Complete Description of the Corresponding Probability Distributions}

Asymptotics for a general case remains a challenge. In the classical statistical approach, it is natural to start with the case of linear functions. Once it is clear how to deal with this case, we can extend our formulas to the case of a general (smooth) function $f\left(x_{1}, \ldots, x_{n}\right)$ : namely, as we have shown in Section 1, we can expand the function $f\left(x_{1}, \ldots, x_{n}\right)$ into Taylor series and use the fact that in a small vicinity of each point, quadratic (and higher order) terms in this expansion can be safely ignored, and we can approximate the original function by the linear terms in its Taylor expansion. In the classical statistical approach, restriction to a small neighborhood makes perfect sense: for example, for a normal distribution, the probability of the deviation $\Delta x$ exceeding six standard deviations $(6 \sigma)$ is so small $\left(\approx 10^{-6 \%}\right)$ that such deviations can be safely ignored. 
In contrast, for a heavy-tailed distribution, the probability density function $\rho(\Delta x)$ decreases slowly with $\Delta x$, as $\rho(\Delta x) \approx A \cdot|\Delta x|^{-\alpha}$. For example, for $\alpha=2$, the probability of $\Delta x$ exceeding $6 \sigma$ is $\approx 6^{-2} \approx 3 \%$, which is quite probable. Even deviations of size $100 \sigma$ are possible: they occur once every 10,000 trials. For such large deviations, we can no longer ignore quadratic or higher order terms; so, we can no longer reduce any smooth function to its linear approximation: each linear function has to be treated separately.

Need to go from asymptotics to a complete description. In the above text, we only find the exponent $\alpha$ corresponding to the asymptotics of the probability distribution for the approximation error $\Delta y=\widetilde{y}-y$. It is desirable to find the whole distribution for $\Delta y$. For that, in addition to the exponent $\alpha$, we also need to find the following:

- the coefficient $A$ at the asymptotic expression $\rho(\Delta y) \sim A \cdot|\Delta y|^{-\alpha}$;

- the threshold $\Delta_{0}$ after which this asymptotic expression provides an accurate description of the probability density, and

- the probability density $\rho(\Delta y)$ on the interval $\left[-\Delta_{0}, \Delta_{0}\right]$ on which the asymptotic expression is not applicable.

Once we know a similar information for the input measurement errors $\Delta x_{1}$ and $\Delta x_{2}$, we can use the formula (3) (or similar formulas corresponding to other data processing algorithms) to estimate the corresponding characteristics for $\Delta y$.

What if we only have partial information about the distribution of errors of direct measurements. In practice, we only have partial information about the probability distributions $\rho_{i}\left(\Delta x_{i}\right)$ of the errors $\Delta x_{i}$ of direct measurements.

Usually, we consider situations in which we know an interval on which the random variable is located with certainty. For example, for normal distribution with mean $\mu$ and standard deviation $\sigma$, we can safely conclude that all possible values are located within the six-sigma interval $[\mu-6 \sigma, \mu+6 \sigma]$, since the probability to be outside this interval is $\leq 10^{-8}$. For such distributions, uncertainty means, e.g., that instead of the exact values of the corresponding cumulative distribution functions $F(x) \stackrel{\text { def }}{=} \operatorname{Prob}(X \leq x)$, we only know an interval $[\underline{F}(x), \bar{F}(x)]$ of possible values of $F(x)$. The corresponding interval-valued function $[\underline{F}(x), \bar{F}(x)]$ is known as a probability box, or p-box, for short; see, e.g., [2,3].

Several algorithms are known for propagating p-boxes via data processing, i.e., for transforming the p-boxes corresponding to the input uncertainty $\Delta x_{i}$ to the p-box for the output uncertainty $\Delta y$. It is desirable to extend these algorithms so hat they will be able to also cover a similar interval uncertainty about the values $A, \alpha$, and $\Delta_{0}$ describing the heavy-tailed distributions.

\section{Acknowledgments}

This work was supported in part by the European Regional Development Fund in the IT4Innovations Centre of Excellence project (CZ.1.05/1.1.00/02.0070), 
by the National Science Foundation grants HRD-0734825, HRD-1242122, DUE0926721, by Grants 1 T36 GM078000-01 and 1R43TR000173-01 from the National Institutes of Health, and by a grant N62909-12-1-7039 from the Office of Naval Research.

The authors are thankful to the anonymous referees for valuable suggestions.

\section{References}

1. Chakrabarti, B. K., Chakraborti, A., Chatterjee, A.: Econophysics and Sociophysics: Trends and Perspectives, Wiley-VCH, Berlin (2006)

2. Ferson, S.: RAMAS Risk Calc 4.0, CRC Press, Boca Raton, Florida (2002)

3. Ferson, S., Kreinovich, V., Hajagos, J., Oberkampf, W., Ginzburg, L.: Experimental Uncertainty Estimation and Statistics for Data Having Interval Uncertainty, Sandia National Laboratories (2007) Publ. 2007-0939.

4. B. Mandelbrot, "The variation of certain speculative prices", J. Business, 1963, Vol. 36, pp. 394-419.

5. Mandelbrot, B.: The Fractal Geometry of Nature, Freeman, San Francisco, California (1983)

6. Mandelbrot, B., Hudson, R. L.: The (Mis)behavior of Markets: A Fractal View of Financial Turbulence, Basic Books, New York (2006)

7. Rabinovich, S.: Measurement Errors and Uncertainties: Theory and Practice, Springer Verlag, New York (2005)

8. Resnick, S. I.: Heavy-Tail Phenomena: Probabilistic and Statistical Modeling, Springer-Verlag, New York (2007)

9. Sheskin, D. J.: Handbook of Parametric and Nonparametric Statistical Procedures, Chapman \& Hall/CRC, Boca Raton, Florida (2007) 